\title{
FLIP-CHIP BASED PACKAGING FOR LINEAR RATCHETING MICROACTUATORS ENABLES 3D STACKS OF MOVEBLE MICROELECTRODES FOR THE BRAIN
}

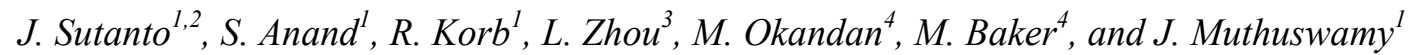 \\ ${ }^{1}$ School of Biological and Health System Engineering, Arizona State University, Tempe, AZ, USA \\ ${ }^{2}$ Amkor Technology, Platform Technology Development, Chandler, AZ, USA \\ ${ }^{3}$ School of Electrical, Computer and Energy Engineering, Arizona State University, Tempe, AZ, USA \\ ${ }^{4}$ Sandia National Laboratories, Albuquerque, NM, USA
}

\begin{abstract}
MEMS based movable microelectrodes now offer the exciting possibility of developing a fully autonomous implantable neural prosthetic system. However, critical interconnect and packaging challenges remain. We report here a novel flip-chip based interconnect and packaging approach for the implantable MEMS microelectrodes with complex moving structures on the die. The proposed approach is chip-scale, versatile (applicable to a variety of substrates and dies), scalable (allowing the realization of 3D stacks), and low-cost. We report here successful in vivo testing of this non-hermetically encapsulated flip-chip based package in long-term rodent experiments.
\end{abstract}

\section{INTRODUCTION}

Emerging prosthetic devices for the brain will enable amputees or patients with peripheral neuropathy, or spinal-cord injuries or stroke to control external robotic devices using their brain signals. The success of brain prosthetic devices is critically dependent on the reliability of implants used to monitor the single neuronal electrical activities in the brain. Several studies have successfully demonstrated the potential of moveable microelectrodes in improving reliability of neuronal recordings by repositioning and seeking new neurons in the event of a failure of the neuron-electrode interface [1-4]. However, the size and weight of the movable microelectrodes are often large, interfering with animal or patient movement/behavior. Therefore, there is a need for a MEMS based movable microelectrode device that can be eventually integrated with advanced signal conditioning and control circuitry towards a fully autonomous neural prosthetic system for the brain. The development of chevron-latch microactuators for movable microelectrodes was reported earlier, where the MEMS chip was packaged on an Au-plated wire bonding package mounted on a $15 \mathrm{~mm}$ x $20 \mathrm{~mm}$ PCB for longterm rodent experiments [5-6]. However, such wire-bonded package and interconnects are bulky, not scalable and cumbersome for implantable applications.

Conventional flip-chip packaging approaches have serious technical problems when applied to MEMS devices with complex moving structures. Additional challenges arise in the case of implantable applications. First, there is a need for high-aspect ratio first-level interconnects (FLI) that will allow sufficient room for the MEMS structures on the die to move and function. Second, flux dissipation in the reflow process is highly uncontrolled resulting in catastrophic contamination of the MEMS structures. Third, even without the underfill, leaks during the encapsulation process can also jam the MEMS structures. Due to the risk of such contamination, the FLI are often moved hundreds of microns away from active MEMS structures increasing the form-factor of the device. We report here a novel low-temperature flip-chip based approach [7-8] using Ag epoxy or solder for FLI. FLI bumps with high aspect ratio $(>2)$ can be made within $70 \mu \mathrm{m}$ from active MEMS structures without any hindrance to MEMS function. The

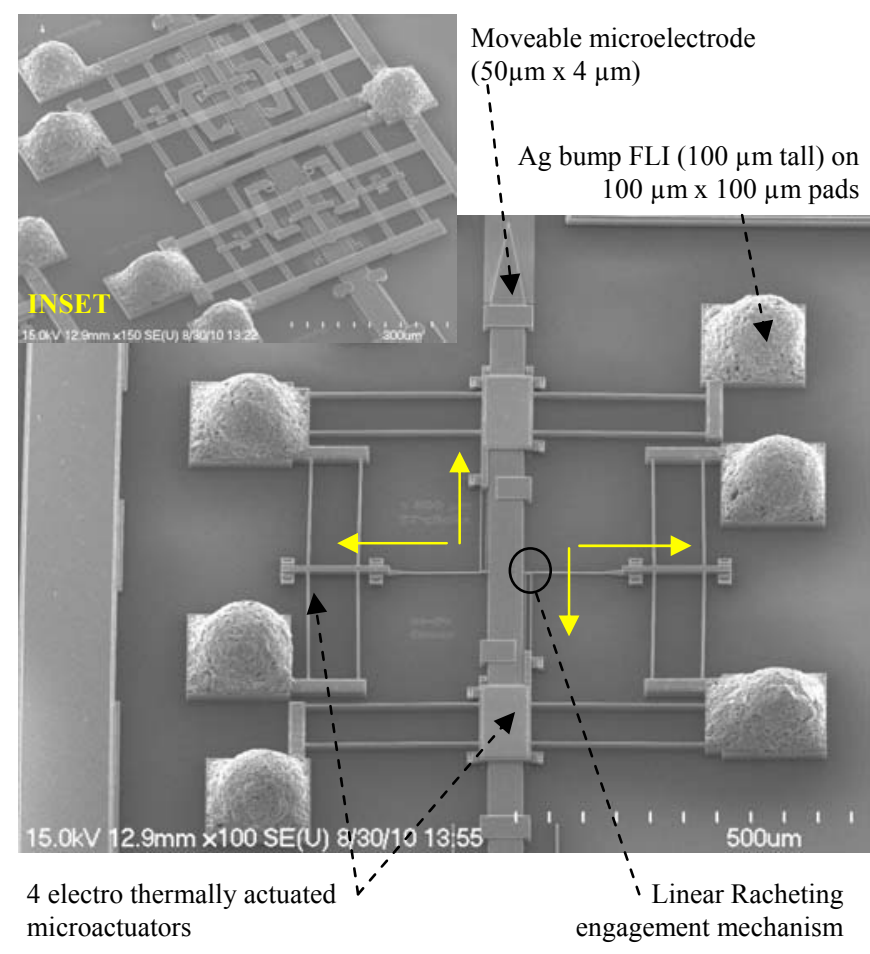

Figure 1. Electro-thermally actuated neural microelectrodes w/ linear ratcheting mechanism. INSET: Previously reported chevron-actuators

flip-chip approach is versatile in that it can be applied to a variety of substrates such as silicon, glass or plastics such as parylene or polyimide.

\section{THEORY}

A. Arrays of MEMS microelectrodes

Arrays of three electrothermally actuated microelectrodes on a 3 $\mathrm{mm} \times 7 \mathrm{~mm}$ chip are microfabricated using SUMMITV ${ }^{\mathrm{TM}}$ process (Sandia Ultraplanar Multi-level Micromachine Technology). In

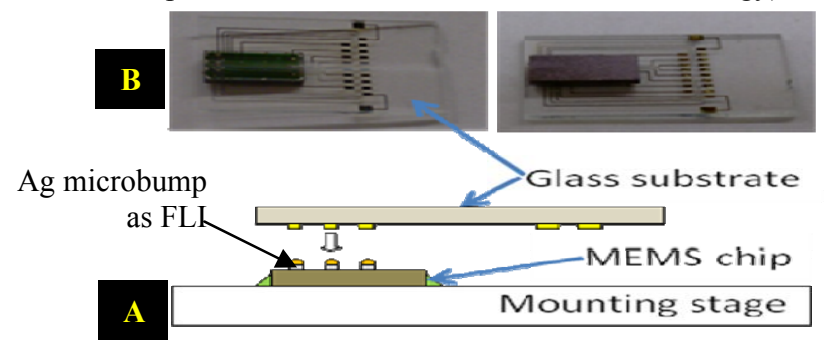

Figure 2. A: Showing the schematic of the flip-chip process where Ag microbumps are used as the FLI. B: MEMS die and glass substrate after flip-chip process

Solid-State Sensors, Actuators, and Microsystems Workshop Hilton Head Island, South Carolina, June 3-7, 2012 
this report, a new generation of MEMS microactuator called the linear ratcheting microactuator is presented as shown in Fig. 1. Compared with the previously reported Chevron-design, the linear ratcheting technique provide a number of advantages, such as higher actuation force $(480 \mu \mathrm{N}$ compared to $220 \mu \mathrm{N})$, smaller actuation step $(6.5 \mu \mathrm{m}$ compared to $8.8 \mu \mathrm{m})$, and more reliable microelectrode movement (both forward and backward).

The current generation of MEMS microelectrode array attempts to address some of the shortcomings of the earlier Chevron-type actuators identified earlier by long-term in vivo testing. The microelectrode has a linear array of teeth spaced 6.5 $\mu \mathrm{m}$ apart at the edges. The 'pawl', a peg-like structure engages the teeth of the electrode and locks it in position, preventing displacement in rest conditions. The new actuators have a simplified movement mechanism with only one 'pawl' that engages with the microelectrode teeth as shown in Fig.1. It has two pairs of electrothermal Chevron actuators that perform identical function. 'Forward drive' actuator and 'disengage forward drive' actuator control the forward movement of the microelectrode and 'reverse drive' and 'disengage reverse drive' actuator control the backward movement of the electrode. The central shuttle of the drive and disengage actuators are both connected in an L-shape arrangement to a 'pawl' that engages the microelectrode. A set of timed pulse waveforms were applied to these actuators to operate the pawl and move the linear ratchet/microelectrode in a given direction.

\section{B. Flip-Chip Packaging technology}

The MEMS die is flip-chipped to the glass substrate via a novel Ag bump FLI as shown in Fig. 2. For more detail on this process, the reader is referred to our earlier report [7-8]. The key steps involved in the current flip-chip process are:

1. Kitting process to prepare the three main components: the MEMS chip, glass substrate, and Omnetics ${ }^{\mathrm{TM}}$ (Omnetics connector corporation) TLI (Third-level interconnects);

2. Bumping process involved dispensing Ag epoxy on each $\mathrm{Al}$ pad to create microscale bumps to build the FLI (First level Interconnects) with a height of about $100 \mu \mathrm{m}$ (total of 18 bumps per testing sample).

3. Flip chip process to a) align the die with respect to the transparent glass substrate, and b) bond the die and substrate

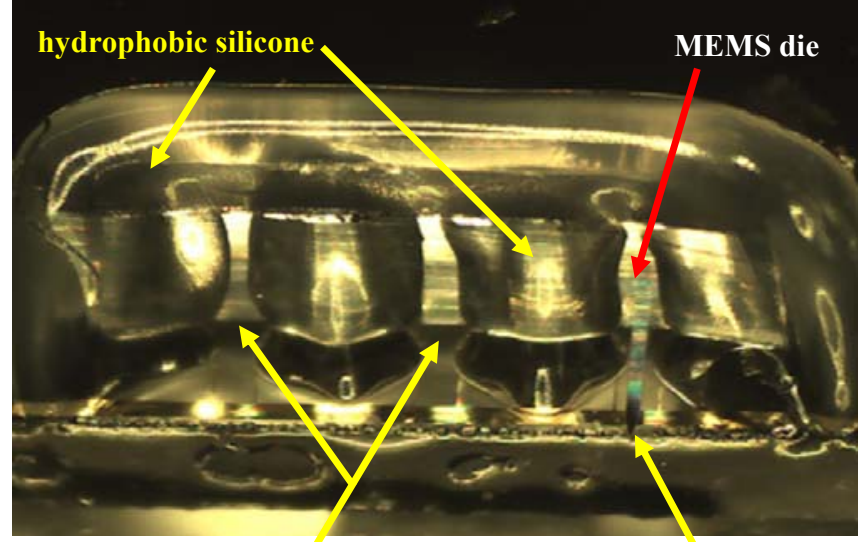

3 of $150 \mu \mathrm{m} \times 100 \mu \mathrm{m}$ hydrophobic micro-channnels

One MEMS microelectrode

$(50 \mu \mathrm{m} \times 4 \mu \mathrm{m})$

Figure 3: Micrograph of the non-hermetic encapsulation (NHE) - with the MEMS die facing down. 3 microchannels each $150 \mu \mathrm{m}(\mathrm{W})$ by $100 \mu \mathrm{m}(\mathrm{H})$ were patterned in hydrophobic silicone to allow egress of microelectrodes. together through the FLI Ag bumps. The sub-steps involved in this process were as follows: (a) the glass substrate was picked up by a suction piston; (b) the MEMS chip located on the chuck was positioned to align it with the glass substrate. To properly bond the FLI bumps the glass substrate was positioned on top of the die with a joint pressure of $20-30 \mathrm{MPa} / \mathrm{bump}$; While the Ag epoxy is still wet (uncured), the chuck was then heated up to $120^{\circ} \mathrm{C}$ for $1 \mathrm{~min}$ to fully cure the Ag epoxy bumps.

4. Connect the TLI to bond the Omnetics ${ }^{\mathrm{TM}}$ connector to the glass substrate by using Ag epoxy. The process was similar to step no 3 .

5. Create non-hermetic encapsulation; In order to create nonhermetic encapsulation on the front side of the die, hydrophobic silicone microstructures were patterned using the dispensing system as shown in the SEM of Fig. 3. A non-hermetic encapsulation (NHE) is important to allow the microelectrodes to move freely inside the brain after implantation and while simultaneously preventing the cerebro-spinal fluids in the brain from entering or contaminating the active MEMS structures. Our previous work achieved a non-hermetic encapsulation by using a screen-mesh with the opening of $60 \mu \mathrm{m}$ coated with silicone gel. In this paper, we report the results of a novel NHE technique compatible with flip-chip process using a non-flow silicone underfill to non-hermetically encapsulate the front edge (from where the microelectrodes emerge before entering the brain) of the integrated package as shown in Fig. 4. Patterns of silicone microstructures were created in the NHE. The $100 \%$ silicone was an auto/marine sealant, clear and commercially available in the hardware store. Pneumatic dispensing technique (EFD Inc.) was used to pattern the silicone microstructures in the NHE on the front edge of the die. The other three edges of the die are nearhermetically sealed using epoxy.

6. Hard protection was a process to apply hard epoxy over the die to protect and introduce a near hermetic seal.

\section{RESULTS}

Compared with the wire-bonding approach reported earlier, the flip-chip technology offered significant reduction in a) the total

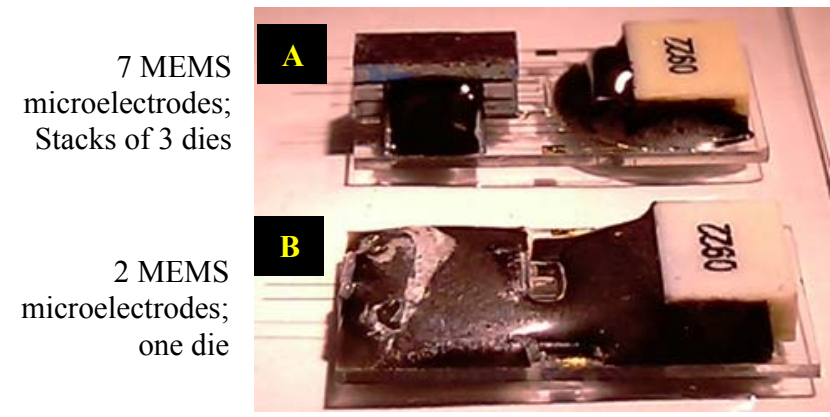

Figure 4. A: Stack of 3 dies, where 7 out of 9 MEMS moveable microelectrodes are actuated B: One die design, where 2 out of 3 MEMS moveable microelectrodes are actuated outside the package boundary

weight of package from 5 grams to 0.5 grams and b) the package thickness from $5 \mathrm{~mm}$ to less than $1 \mathrm{~mm}$. One of the advantages of flip-chip packaging is the ability to scale up the number of chips without significantly increasing the X-Y form factor by facilitating 3D stacks. The flip-chip technology is extended to realize a chipscale 3D stack using flip-chip technology and packaging processes. A 3D flip-chip stack of 3 MEMS microactuator dies is shown in Fig. 4A. Such 3D stacks enable the development of high-density movable microelectrodes that enable the monitoring of ensemble of neurons. For prosthetic applications, higher degree of freedom 


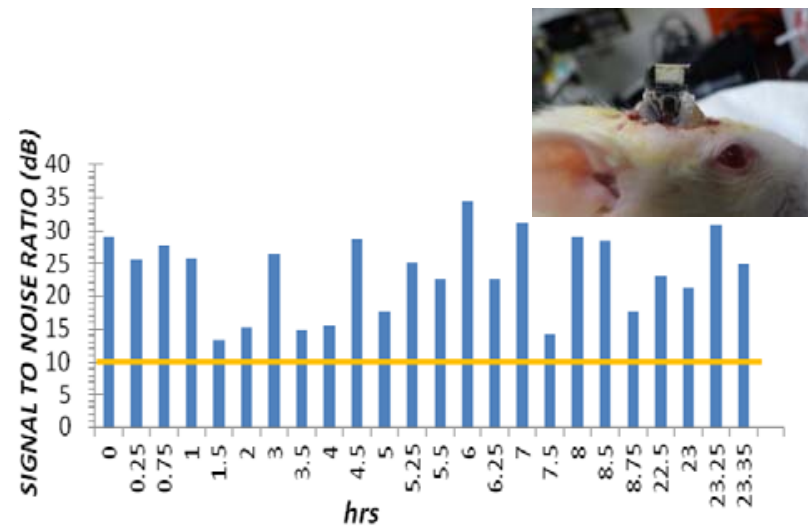

Figure 5: SNR (signal-to-noise ratio) over $24 \mathrm{hrs}$ of continuous neural recordings from rodents. SNR is always above the 10 $\mathrm{dB}$ threshold that corresponds to units that are barely discernible.

and precision can arguably be achieved by sampling larger numbers of neurons.

The packaged MEMS moveable electrodes were surgically implanted in the rodent brain. The packaged device is mounted on the rodent skull. Four mounting screws are initially attached to the bone/skull surrounding the perimeter of the craniotomy, and the microelectrodes are implanted in the motor cortex. After opening the skull, the dura is carefully resected; followed by introducing gel foam on the perimeter of the craniotomy. Silicon microchannels were patterned inside the mould cavity with three holes at the same spacing as the ones for MEMS microelectrodes. A small spacer (made of silicone) was then made between the skull and the MEMS package to accommodate the curvature of the skull. By using stereotactic equipment, the front side of the MEMS package is brought in contact with the spacer, followed by placing dental cement surrounding the package. The dental cement is anchored to the screws. A test under pressurized liquid immersion was done on the MEMS package after SHS was applied to determine its break-down pressure, which was found to be $80 \mathrm{~cm}$ of water. The pilot in vivo test of NHE implanted on the rodents' skull shows no liquid infiltration after implant for more than 77 days. The test eventually failed due to cracks in the glass substrate

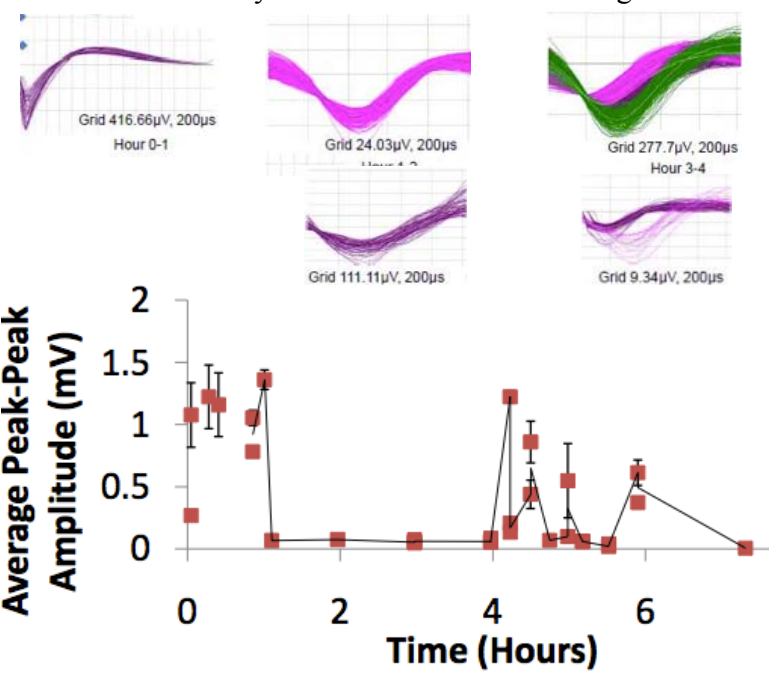

Figure 6: Trend in average peak-to-peak amplitudes in the neural action potentials over the first 7 hours are plotted with typical action potentials observed at different time points shown above. while no damage was observed in the NHE. Figure 5 shows the signal-to-noise ratio (SNR) of neural recording from one MEMS microelectrodes over 24 hours of nearly continuous recording immediately after surgery. SNR is over $10 \mathrm{~dB}$ - which corresponds to the threshold where the action potential waveforms are barely discernible. The peak-to-peak amplitudes of single unit activities over 7 hours on a free behaving rodent are shown in Fig. 6.

\section{CONCLUSIONS}

A novel flip-chip based packaging approach for MEMS devices was developed and was successful tested for monitoring single neuronal activity in long-term rodent experiments. The proposed approach is a low-temperature process that is applicable to silicon, glass or plastic substrates and also a variety of MEMS devices that involve complex moving structures on their die. The non-hermetic encapsulation was found to be adequate in keeping the brain fluids out of the MEMS device over a period of 77 days The interconnects were successfully tested by obtaining continuous neural recording over a period of $24 \mathrm{hrs}$ immediately after implantation. The flip-chip interconnects through Ag bumps provided chip-scale interconnects that allowed for the development of 3D stacks of MEMS dies. A stack of 3 MEMS dies was developed that was mechanically stable. The electrical interconnects for the stack is a work in progress.

\section{ACKNOWLEDGEMENTS}

We would like to thank NIH (NS055312-S1) for funding this research and the Center for Solid State Electronic Research (CSSER) at Arizona State University for use of their clean room facilities.

\section{REFERENCES}

[1] S. Chakrabarti, P. Hebert, M. T. Wolf, M. Campos, J. W. Burdick, and A. Gail, "Expert-like performance of an autonomous spike tracking algorithm in isolating and maintaining single units in the macaque cortex," $J$ Neurosci Methods, vol. 205, pp. 72-85, 2012.

[2] J. G. Cham, E. A. Branchaud, Z. Nenadic, B. Greger, R. A. Andersen, and J. W. Burdick, "Semi-chronic motorized microdrive and control algorithm for autonomously isolating and maintaining optimal extracellular action potentials," J Neurophysiol, vol. 93, pp. 570-9, 2005.

[3] M. S. Fee and A. Leonardo, "Miniature motorized microdrive and commutator system for chronic neural recording in small animals," J Neurosci Meth, vol. 112, pp. 83-94, Dec 152001.

[4] A. Jackson and E. E. Fetz, "Compact movable microwire array for long-term chronic unit recording in cerebral cortex of primates," J Neurophysiol, vol. 98, pp. 31093118, 2007.

[5] N. Jackson, A. Sridharan, S. Anand, M. Baker, M. Okandan, and J. Muthuswamy, "Long-Term Neural Recordings Using MEMS Based Movable Microelectrodes in the Brain," Front Neuroeng, vol. 3, p. 10, 2010.

[6] J. Muthuswamy, M. Okandan, A. Gilletti, M. S. Baker, and T. Jain, "An array of microactuated microelectrodes for monitoring single-neuronal activity in rodents," IEEE T Bio-med Eng, vol. 52, pp. 1470-7, Aug 2005.

[7] J. Sutanto, S. Anand, C. Patel, and J. Muthuswamy, "Novel First-Level Interconnect Techniques for Flip-chip on MEMS devices " J Microelectromech S, vol. 21, pp. 132144,2012 
[8] J. Sutanto, S. Anand, A. Sridharan, R. Korb, L. Zhou, M. Baker, M. Okandan, and J. Muthuswamy, "Packaging and Non-Hermetic Encapsulation Technology for Flipchip on Implantable MEMS Devices," $J$ Microelectromech $S$, vol. in press, 2012.

\section{CONTACT}

Jit Muthuswamy

Associate Professor, Biomedical Engineering

ECG 334, P.O. Box 879709

School of Biological and Health Systems Engineering

Arizona State University

Tempe, AZ 85287-9709

Ph: (480) 9651599

jit@asu.edu 